\title{
EDITORIAL
}

\section{Neuroimaging: Anything to Do with Neurotherapeutics?}

\section{INTRODUCTION}

Neuroimaging in neurotherapeutics? It seems a contradiction in terms. Traditionally, the management of a patient has consisted of two sequential processes: 1) diagnosis, and 2) therapy. Of course, choosing the appropriate therapy depends on the correct diagnosis, but then therapy proceeds on its own terms, addressing the responsible malady. Neuroimaging has traditionally fallen squarely in the diagnostic stage. Not any more. As this issue of NeuroRx ${ }^{\circledR}$ proves, neuroimaging is now a powerful tool in neurotherapeutics. It facilitates and, indeed, enables the development of new therapies, allows for more efficient testing of their applicability in clinical trials, and is useful for monitoring their effect on the nervous system.

Remedies have become available in the past two decades for many neurological diseases previously untreatable such as Alzheimer's disease and multiple sclerosis (MS). ${ }^{1,2}$ Currently, detailed knowledge on the genetics and biochemistry underlying a number of neurological diseases ushers an ever quickening pace of therapeutic discovery and clinical testing. ${ }^{3}$ To test therapeutic effectiveness, in experimental animals as well as in humans, measuring the longitudinal response to a new therapy is essential. For this purpose, longitudinal biomarkers are needed. Some neurological diseases, such as Parkinson's and Alzheimer's, begin much before they are clinically symptomatic. ${ }^{4}$ Thus, ideal longitudinal markers should detect disease-related changes in the presymptomatic stage, when therapies that would stem or reverse the process could preclude neuronal loss or other form of tissue damage leading to the development of symptoms. For many neurological disorders, neuroimaging meets the requirements of an ideal biomarker. The neuroimaging characteristics of a given disorder are often effective markers of 1) the predisposition to develop a disorder in a clinically healthy individual, 2) the onset of disease, and 3) its rate of progression. In a recent review in NeuroR $x^{\circledR}$, Fox and Growdon have referred to these three stages as 1) trait, 2) state, and 3) rate..$^{5}$

\section{NEUROIMAGING IN EXPERIMENTAL ANIMAL MODELS}

Neuroimaging is an effective tool to test disease mechanisms and potential therapeutic agents in animal models. ${ }^{6,7}$ Optical technologies, magnetic resonance imaging
(MRI) and spectroscopy, molecular imaging by positron emission tomography (PET) or single-photon emission tomography (SPECT), and other technologies are assisting in moving CNS drug development from in vitro biology to in vivo integrative mammalian biology of disease. ${ }^{6}$ For instance, micro-PET, with a resolution of approximately $1.5 \mathrm{~mm}$, allows for the study of gene and protein expression, and other mechanisms of disease in rat models. In a micro-PET study, Sanchez-Pernaute et al. ${ }^{8}$ studied in vivo the effect of a selective inhibitor of the inducible form of cyclooxygenase (COX-2) on a rat model of Parkinson's disease (PD). Nigral neuron degeneration was induced by the intrastriatal injection of 6-hydroxydopamine. The inflammatory response was monitored by micro PET with ${ }^{11} \mathrm{C}-\mathrm{PK} 11195$ (N-secbutyl-1-(2-chlorophenyl)-N-methylisoquinoline-3-carboxamide), a benzodiazepine receptor ligand and specific marker for activated macrophages/microglia. The loss of dopaminergic neurons was monitored with ${ }^{11} \mathrm{C}$-CFT $[2 \beta$ carbomethoxy-3 $\beta$-(4-fluorophenyl) tropane], a specific ligand for presynaptic dopamine (DA) transporters. Between 12 and 21 days, there was a significant progression of inflammation and DA cell loss in the vehicle group, both of which were prevented by the COX-2 inhibitor. A similar imaging paradigm can then be applied to humans to study similar pathogenetic and therapeutic mechanisms. As can be appreciated from this example, what is critical is that the animal model may resemble closely the human disease. It may mimic all the abnormal molecular mechanisms of the human disease or, more often, one of them.

Using optical imaging, researchers can assess the effect of gene therapy or implanted neural precursor stem cells (NPCs) in animal models of glioblastoma multiform, a malignant brain tumor with a high regrowth rate after surgical resection or radiation therapy. NPCs bearing a tumor-control protein, S-TRAIL (secreted tumor necrosis factor-related apoptosis-inducing ligand), were engineered with luminescent and fluorescent transgenes (Fluc) for fluorescence and dual bioluminescence imaging. ${ }^{9}$ With a highly malignant human-glioma rat model expressing Renilla luciferase, intracranially implanted NPCs expressing both Fluc and S-TRAIL were shown to migrate into the tumors and have antitumor effects. ${ }^{9}$ The design of small tumor-specific antibody fragments is another attractive way for the specific detection of tumor cells by imaging in vivo as well as for targeted radioimmunotherapy. ${ }^{10}$ 
The brains of mice, more readily available than rats as disease models with knockout genes or transgenes, are too small to be imaged by micro-PET but can be imaged with ultra high-field MRI. A marker of Alzheimer disease, amyloid plaque density can be visualized and quantitated without the injection of contrast material in a 9.4 Tesla MRI unit. ${ }^{11}$ With this model, statins, nonsteroidal antiinflammatory agents and other small molecules with the potential of lowering amyloid plaque deposition can be readily tested.

This drug discovery approach involves the production of an animal model, the characterization of molecular abnormalities likely to be present in the human disease, the identification of an imaging protocol to measure those abnormalities and the production of small molecules that can correct them. In the case of PET and SPECT, the same small molecules can be used as diagnostic markers at small doses and as therapeutic agents at larger doses.

As Phelps points out, ${ }^{6}$ PET academic centers and industry are collaborating to couple diagnostic radiopharmaceuticals (i.e., molecular imaging probes) and therapeutic pharmaceuticals, thus combining the goals of molecular diagnostics and molecular therapeutics. Molecules likely to be effective in an animal model and their analogs are tested by neuroimaging: molecular imaging probes in low doses to image and measure the target function, and then the dose of the molecule is increased to pharmacologic levels to modify the target function. Ideal molecular imaging probes and drugs share several properties: small molecule; high affinity for target and low affinity for nontargets; sufficient lipophilicity or carrier system to cross cell membranes; low peripheral metabolism. Desired properties differ in that imaging probes but not drugs need a target to background affinity greater than one, and plasma clearance times of minutes to hours are desired for imaging, whereas hours to days are preferred for therapeutic drugs.

Together with more traditional biological or behavioral measurements, imaging is used to evaluate molecular imaging probes and labeled drugs first in mice or rats, after which similar studies are repeated in a small number of patients to assess their similarity to the experimental animal situation. The animal models facilitate the process of titrating drug to disease target in tissue for accurate dosing, using a labeled form of the drug. Together with imaging in humans, animal imaging can be used to determine the pharmacodynamic and pharmacokinetic properties of drugs and probes and whether the drug tested restores to normal the mechanisms affected by the disease process.

If small studies are positive, larger animal studies are performed to prepare larger studies in humans. Thus, biological scientists, academic physicians, and those in the pharmaceutical industry work together in moving knowledge and applications from the basic level to patients with a better scientific foundation. This leads to relatively low cost rapid screening and elimination of ineffective compounds, the overwhelming majority of those tested.

\section{NEUROIMAGING IN CLINICAL TRIALS}

Neuroimaging is useful to define the biological mechanisms of human disease, its onset and progression (trait, state and rate). In the presymptomatic stage (trait), neuroimaging findings can help guide genetic testing. For instance, the yield of the determination of the CADASIL gene will be higher if focused on individuals who have migraine and specific white matter changes on MRI. ${ }^{12}$ Susceptibility genes or single nucleotide polymorphisms for Alzheimer's disease are likely to be more frequent in elderly populations with lower metabolism on PET in the inferior parietal and posterior cingulate regions of both hemispheres. ${ }^{13}$

For the selection of adequate patient samples, the clinical phenotype often lacks specificity. Clinical trials of MS, for instance, require MRI to identify the characteristic white matter lesions. ${ }^{14}$ Neuroimaging is often essential to define the presence of the disease (state), as in the case of brain tumors or MS. ${ }^{10,14}$ What is so obvious for these diseases, applies to the disorders that, as examples of major neurological disease categories, are discussed in this issue of NeuroRx ${ }^{\circledR}$. The individual subject with a well-defined neurological disease can then be enrolled in a clinical study to evaluate the effectiveness of a new therapeutic approach.

Even with characteristic phenotypes, such as PD, imaging can help identify more homogeneous patient samples, rendering the therapeutic trial more reliable and less expensive. Imaging was used in two therapeutic trials of PD. ${ }^{15}$ In the REAL PET study, 21 out of 183 recruited cases $(11 \%)$ clinically thought to have PD had a normal ${ }^{18}$ F-dopa PET. ${ }^{16}$ Similarly, in the ELLDOPA trial, 21 out of 135 cases $(16 \%)$ felt to have PD had normal ${ }^{123} \mathrm{I}-\beta$ SPECT. ${ }^{17}$ These subjects have now been followed for up to 6 years, and both their clinical syndromes and imaging findings are unchanged. ${ }^{15}$ It is likely that their disorder differs from the neurodegenerative process of the patients with progression, and therefore they are not good candidates to test neuroprotective therapies. Either these patients are excluded by imaging dopaminergic function at baseline or one should allow for a $10-15 \%$ discordance between clinical impression and PET or SPECT assessments of striatal dopaminergic function when powering trials to show a given effect size. ${ }^{15}$

In the longitudinal evaluation of the effect of a drug, neuroimaging has great potential. For instance, in a study of a new muscarinic agonist for Alzheimer's disease with a sample size of 192 patients and a follow up of 1 year, 
disease progression was better gauged in $99 \%$ of the patients with measurements of hippocampal atrophy on MRI than with cognitive or behavioral testing $(p<$ 0.001). ${ }^{18}$ Using neuroimaging markers would allow for a marked reduction of sample size. In that study, the estimated number of subjects per arm required to detect a $50 \%$ reduction in the rate of decline over 1 year was as follows: AD Assessment Scale-cognitive subscale, 320; Mini-Mental Status Examination, 241; hippocampal volume, 21; and temporal horn volume, 54. ${ }^{18}$ Although the efficacy of disease-modifying treatments for Alzheimer's disease must ultimately be demonstrated using clinically meaningful outcome measures such as the slowing of decline in cognitive function, such trials will likely require hundreds of patients studied for a minimum of $1-2$ years. ${ }^{19}$ Thus, neuroimaging as a surrogate marker of efficacy, with less variability than clinical assessments, can be extremely useful to reduce the number of subjects. Particularly in the early phases of clinical drug testing, it may provide proof of principle of drug safety and efficacy.

PET and other neuroimaging techniques have the potential, still unrealized, to be of great assistance in the important field of pharmacogenetics. The efficacy and side effects of a drug depend on the specific receptor configuration of the individual patient. PET tracers can provide receptor and enzyme active site dose occupancy profiles, guiding dosage selection for phase I and phase II trials. ${ }^{15}$ Providing a surrogate marker for drug efficacy, neuroimaging could advance the concept of personalized medicine where receptor and enzyme binding profiles help predict therapeutic outcome. ${ }^{15}$

\section{THIS ISSUE OF NeuroRx ${ }^{\circledR}$}

This issue of NeuroRx ${ }^{\circledR}$ offers a review of the use of imaging in neurotherapeutics. Because the methodology of imaging is complex, this review has two parts. Part I focuses on the techniques used for neuroimaging in neurotherapeutics. Part II conveys information on the application of neuroimaging to a number of common disorders of the nervous system.

In Part I, authors with extensive experience in each technology explain its usefulness in neurotherapeutics. As the reader will notice, the authors tend to be sanguine about the technique they discuss. Knowing it best and having had a positive experience with its effectiveness in the therapy of neurological disorders, they may extol it as the most useful for this purpose. Because the few headto-head studies comparing the usefulness of these techniques for a given disorder are discussed in Part II, the editors have chosen to spare the individual emphasis and let the readers draw their own conclusions from the evidence shown by the authors.

\author{
Jose C. Masdeu* and Rohit Bakshi ${ }^{\dagger}$ \\ Guest Editors \\ *Departments of Neurology and Neurosurgery \\ University of Navarra Medical School \\ Clínica Universitaria de Navarra and \\ Center for Applied Medical Research \\ 31008 Pamplona, Spain \\ E-mail: masdeu@unav.es \\ and \\ †Departments of Neurology and Radiology \\ Center for Neurological Imaging \\ Harvard Medical School at the \\ Brigham \& Women's Hospital \\ Boston, Massachusetts 02115 \\ E-mail: rbakshi@bwh.harvard.edu
}

Acknowledgments: This work was supported by the UTE Fundación para la Investigación édica Aplicada (Foundation for Applied Medical Research), Pamplona, Spain, and by research grants from the Spanish Ministry of Health (FIS 01/ 10009, to J.M.), from the National Institutes of Health (NIHNINDS NS42379, to R.B.) and the National Multiple Sclerosis Society (RG 3574A1, R.B.).

\section{REFERENCES}

1. Cummings JL. Alzheimer's disease. N Engl J Med 351:56-67, 2004.

2. Weinstock-Guttman B, Bakshi R. Combination therapy for multiple sclerosis: the treatment strategy of the future? CNS Drugs 18:777-792, 2004.

3. Hardy J. Toward Alzheimer therapies based on genetic knowledge. Аппи Rev Med 55:15-25, 2004.

4. DeKosky ST, Marek K. Looking backward to move forward: early detection of neurodegenerative disorders. Science 302:830-834, 2003.

5. Fox N, Growdon JH. Biomarkers and surrogates. NeuroRx 1:181, 2004.

6. Phelps ME. Inaugural article: positron emission tomography provides molecular imaging of biological processes. Proc Natl Acad Sci USA 97:9226-9233, 2000

7. Pirko I, Fricke ST, Johnson AJ, Rodriguez M, Macura SI. Magnetic resonance imaging, microscopy, and spectroscopy of the central nervous system in experimental animals. NeuroRx 2:250264, 2005.

8. Sanchez-Pernaute R, Ferree A, Cooper O, Yu M, Brownell AL, Isacson O. Selective COX-2 inhibition prevents progressive dopamine neuron degeneration in a rat model of Parkinson's disease. J Neuroinflammation 1:6, 2004.

9. Shah K, Weissleder R. Molecular optical imaging: applications leading to the development of present day therapeutics. NeuroRx 2:215-225, 2005.

10. Jacobs AH, Kracht LW, Gossmann A, Rüger MA, Thomas AV, Thiel A, Herholz K. Imaging in neurooncology. NeuroRx 2:333$347,2005$.

11. Jack CR Jr, Garwood M, Wengenack TM, Borowski B, Curran $\mathrm{GL}$, Lin J, et al. In vivo visualization of Alzheimer's amyloid plaques by magnetic resonance imaging in transgenic mice without a contrast agent. Magn Reson Med 52:1263-1271, 2004.

12. Gladstone JP, Dodick DW. Migraine and cerebral white matter lesions. When to suspect cerebral autosomal dominant arteriopathy with subcortical infarcts and leukoencephalopathy (CADASIL). Neurologist 11:19-29, 2005.

13. Small GW, Ercoli LM, Silverman DH, Huang SC, Komo S, Bookheimer SY, et al. Cerebral metabolic and cognitive decline in persons at genetic risk for Alzheimer's disease. Proc Natl Acad Sci USA 97:6037-6042, 2000. 
14. Bakshi R, Minagar A, Jaisani Z, Wolinsky JS. Imaging of multiple sclerosis: role in neurotherapeutics. NeuroRx 2:277-303, 2005.

15. Brooks DJ. Positron emission tomography and single-photon emission computed tomography in central nervous system drug development. NeuroRx 2:226-236, 2005.

16. Whone AL, Watts RL, Stoessl AJ, Davis M, Reske S, Nahmias C, et al. Slower progression of Parkinson's disease with ropinirole versus levodopa: the REAL-PET study. Ann Neurol 54:93-101, 2003.
17. Parkinson Study Group. Does levodopa slow or hasten the rate of progression of Parkinson disease? The results of the ELLDOPA trial. Neurology 60(Suppl 1):A80-A81, 2003.

18. Jack CR Jr, Slomkowski M, Gracon S, Hoover TM, Felmlee JP, Stewart K, et al. MRI as a biomarker of disease progression in a therapeutic trial of milameline for AD. Neurology 60:253-260, 2003.

19. Dickerson BC, Sperling RA. Neuroimaging biomarkers for clinical trials of disease-modifying therapies in Alzheimer's disease. NeuroRx 2:348-360, 2005. 